\title{
mouvements liés à des travaux de défense et de restauration des sols
}

\author{
par \\ J.P. Flotte \\ Institut National Agronomique, Alger
}

RESUME - L'implantation de banquettes d'infiltration est l'élément principal des travaux de défense et de restauration du sol en Algérie.

Les travaux de D.R.S. ont pour but de freiner l'érosion, mais là où le reboisement est absent ou insuffisant les banquettes induisent des mouvements du sol. Ia technique des banquettes n'est pas condamnable en bloc, mais elle a été utilisée sans discernement, d'autre part leur conception ne repose que sur la valeur des pentes, or nos observations font apparaître que ce principe doit être rejeté lorsque certains facteurs se trouvent réunis : une lithologie "très sensible", la présence de glissements, une pluviométrie importante.

\section{INTRODUCTION}

Les travaux de défense et de restauration du sol ont conmencé en Algérie en 1942, et se poursuivent de nos jours. Le but principal des travaux est, en freinant l'érosion, de diminuer le débit solide des oueds et d'éviter ainsi l'envasement des barrages. Mais seules ont été boisées les banquettes des terrains domaniaux et les parties les moins peuplées des bassins versants; ailleurs des mouvements du sol sont apparus. Le système des banquettes a déjà fait l'objet de critiques pour d'autres régions du Maghreb, nous décrirons quelques cas typiques observés dans I'Atlas tellien de la région d'Alger.

\section{CONCEPTION ET BUT DES BANQUETTES}

Les calculs utilisés pour la mise en place des banquettes s'appuient sur un critère unique : la pente (Saccardy-1950) .

Il n'est question ici que des banquettes à profil déversé vers l'amont et des banquettes à profil normal, réservées aux pentes supérieures à 10 \%, les banquettes à profil amorti à double courbure ne concernent pas la région du Tell. Les banquettes devaient présenter de multiples avantages : défense contre les processus d'érosion, cicatrisation rapide des griffes et des ravines, reprise de la génèse pédologique, augmentation générale de l'infiltration, diminution des dépôts torrentiels, atténuation des crues, diminution du débit solide, relèvement du niveau des nappes souterraines, régulation du régime des oueds, rétablissement des pentes d'équilibre, augmentation de la production sylvo-pastorale et agricole.

\section{OBSERVATIONS.}

Les banquettes ont donné de bons résultats sur les pentes modérées, ainsi que là où la mise en place s'est accompagnée d'un reboisement. En revanche, sur les pentes fortes non boisées ou cultivées, les banquettes n'ont pas donné les résultats escomptés, et des désordres sont apparus.

Les mouvements du sol sont de divers types et souvent même composites.

reptation, solifluxion et battance sur la banquette

Ia reptation affecte principalement le fond sous-solé des banquettes à profil nonmal qui est légèrement déversé vers l'amont. Elle se manifeste par une série d'ondulations à peine marquées. Des piquets térmoins se sont déplacés de 3 à $5,5 \mathrm{~cm}$ pendant la saison pluvieuse (Octobre 1978 à Avril 1979), la profondeur maximale observée est de $7 \mathrm{~cm}$. Les analyses sédimentométriques ont montré que 80 \% des éléments étaient de la taille des limons et des sables fins $(2$ à $100 \mu)$.

La solifluxion affecte les pentes des bourrelets, elle se manifeste par des ondulations larges de 30 à $50 \mathrm{~cm}$, la vitesse du mouvement est de $12 \mathrm{~cm}$ par an (1979), 85 \% des éléments ont une taille inférieure à $20 \mu$ (limons et argiles).

Le phénomène de battance se manifeste sur le fond des banquettes, qui est parsemé de fentes de dessication à la fin de l'été, lors des premières pluies les éléments fins désagrégés sont entraînés dans les fissures et les colmatent, l'infiltration ne se fait plus, il en résulte la formation d'une "semelle saturée" glissant sur un substratum peu perméable. 
L'eau ne s'infiltrant plus ruisselle en nappe sur le fond de la banquette, se concentre au pied du talus et en incise le pied; le talus sapé à sa base glisse.

Reptation, solifluxion et battance sont provoquées par la destruction de la chaîne des sols lors de l'édification des banquettes; à la place d'horizons structurés et cohérents, il ne reste qu'un maigre manteau d'altération remanié !

Ces phénomènes sont mineurs et superficiels, mais à moyen terme ils entraînent la disparition du bourrelet par tassements successifs, et le glissement du talus par sapement à la base (figure 1) .

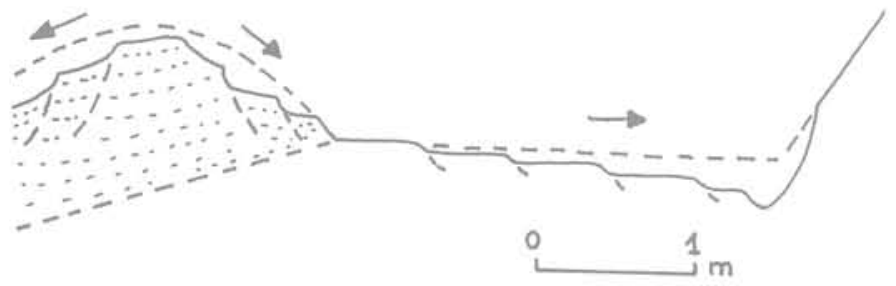

Fig. 1 - Coupe d'une banquette à profil normal affectée par la reptation et la solifluxion avec incision au pied du talus (environs de Tablat).

\section{glissement du talus et du bourrelet de la banquette}

Le talus fait de matériel remanié glisse sur le substratum (figure 2).

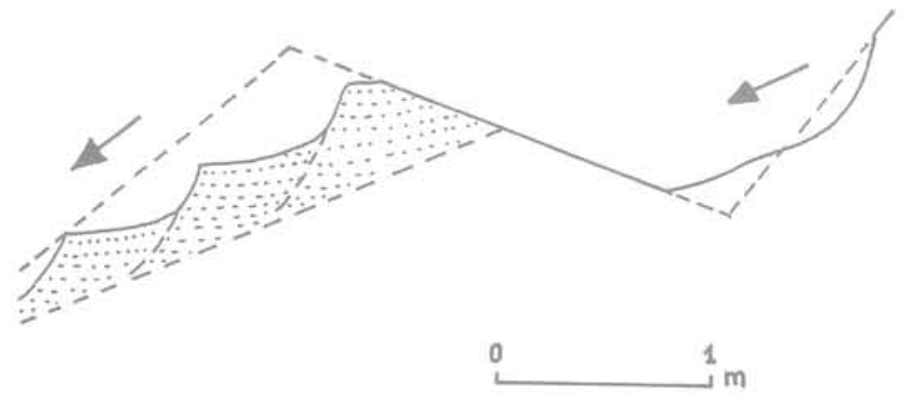

Fig. 2 - Coupe d'une banquette à profil déversé dont le bourrelet et le talus sont affectés par des glissements (environs de Tablat).
Le glissement du talus a pour cause sa pente excessive $(100$ \%) dans un matériau (marnes et flyschs altérés) dont la pente limite de stabilité naturelle est tout au plus de $80 \%$ dans le meilleur des cas. Le glissement du talus bloque 1 'évacuation des eaux, vers l'exutoire, l'eau s'accumule derrière le bourrelet, s'infiltre dans celui-ci et finit par provoquer son glissement.

Les glissements entraînent la destruction de la banquette d'autant plus facilement qu'ils facilitent l'érosion par ruissellement (figure 3).

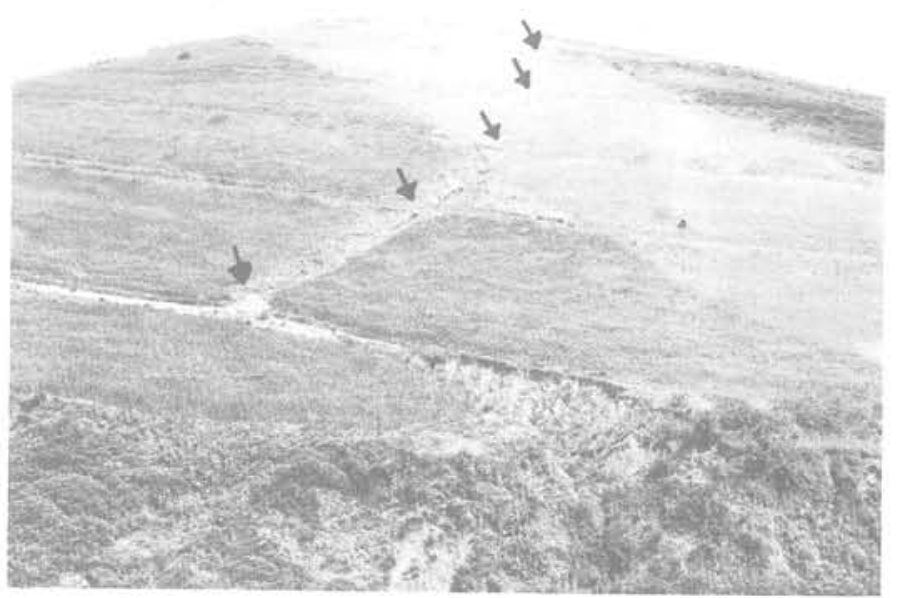

Fig. 3 - Coupure des banquettes par glissement du talus et du bourrelet, puis ravinement. Le phénomène a débuté au bas de la pente, puis par érosion régressive a gagné en amont ; la banquette la plus haute est à peine entamée (Borj Menaĩel).

$\mathrm{NB}$ - remarquer le glissement du substratum sur la droite, et la solifluxón généralisée du versant, les ondulations sont bien visibles sur la partie gauche de la photographie.

\section{glissement de la banquette et du versant}

Ce type de glissement est provoqué par l'infiltration des eaux dans le versant argileux. Le bourrelet (ou le talus) glisse avec le substratum (figure 4). Ie ravinement s'installe dans la niche et le corps du glissement, entrainant la destruction de la banquette, puis de I'interbanquette par érosion régressive et glissement avec sapement et appel au vide. 


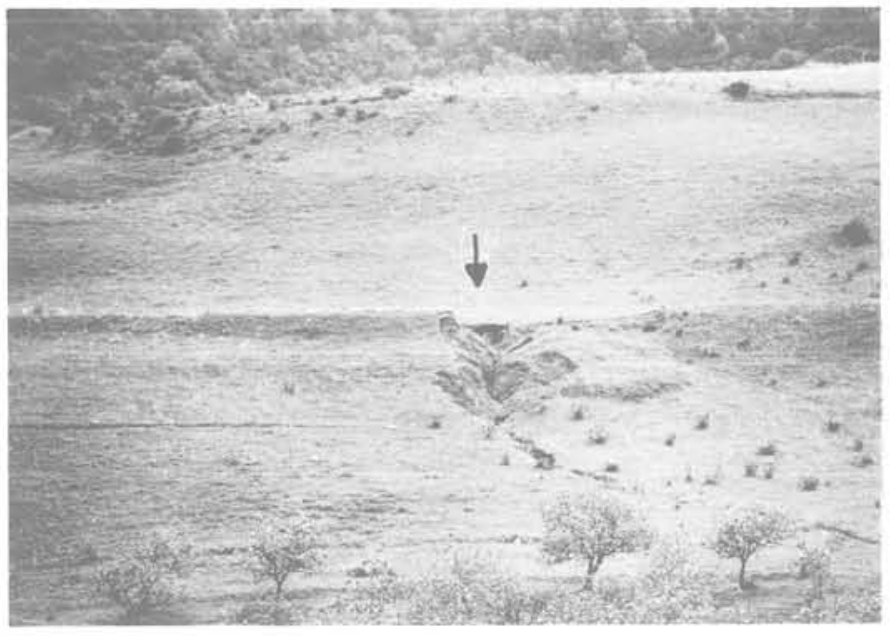

Fig. 4 - Glissement du bourrelet et de son substratum (Bordj Menaīel).

$\mathrm{NB}$ - remarquer le ravinement important dans le glissement et les ondulations importantes révélant l'instabilité du versant.

\section{mouvements du sol induits par l'évacuation des eaux}

Le débouché des banquettes sur l'exutoire est le point le plus fragile de l'ouvrage, trop souvent non aménagé il est alors rapidement raviné, le bourrelet sapé à la base glisse, la ravine s'élargit, s'approfondit et gagne vers l'amont, elle finit par saper le talus, puis l'interbanquette, d'année en année le ravinement progresse vers l'amont (figure 5) .

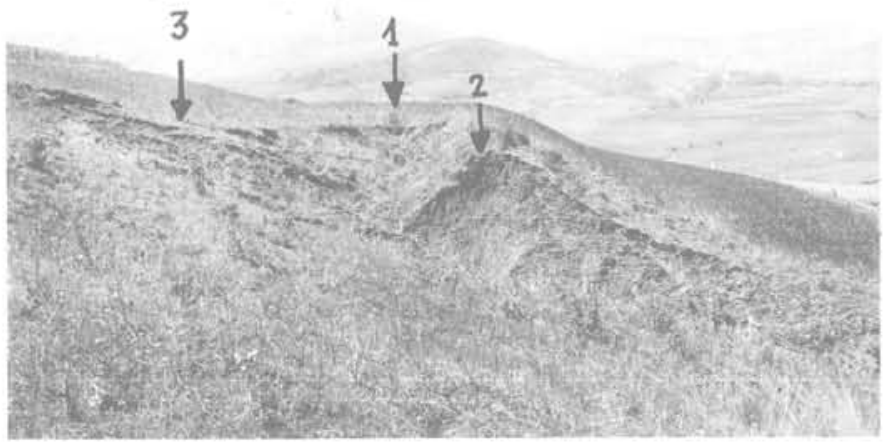

Fig. 5 - 1-Ravinement à l'embouchure d'une banquette 2-Sapement latéral du bourrelet.

3-Sapement du talus puis de I'interbanquette par érosion régressive (Bordj Menaīel).

Parfois l'exutoire est sur un ancien glissement qui se trouve réactivé, le fond de l'exutoire s'effondre par soutirage, tandis que les eaux s'infiltrent profondément et créent un glissement de grande ampleur (figures 6 et 7 ).

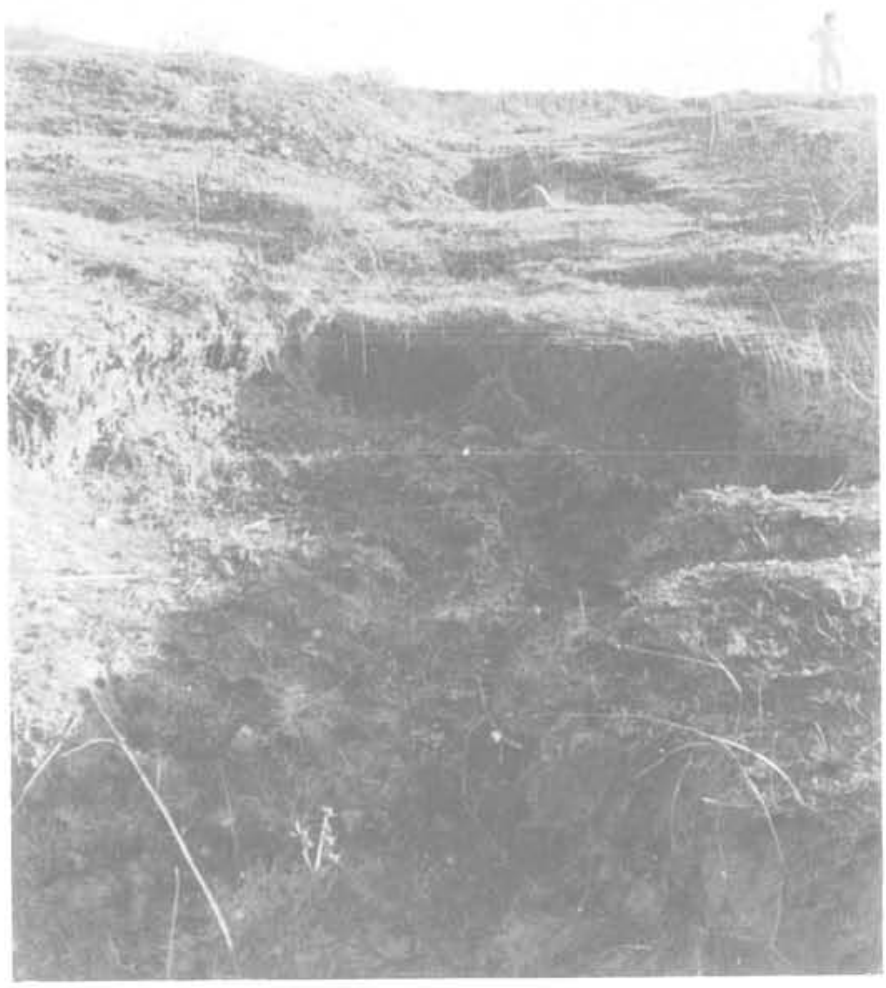

Fig. 6 - Soutirage dans un exutoire.

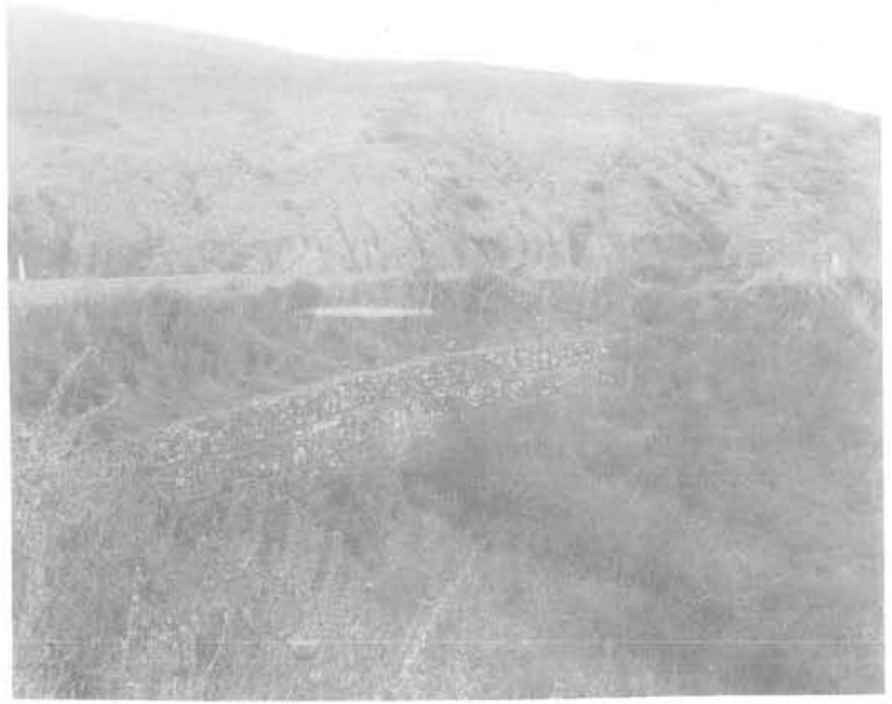

Fig. 7 - Glissement provoqué par l'infiltration des eaux dans la partie amont d'un exutoire.

\section{IDENTIFICATION MINERALOGIQUE ET GEOTECHNIQUE DES} MATERTAUX

L'examen de la fraction argileuse au microscope électronique à transmission a révélé la présence de quatre types d'argile : montmorillonite, illite, kaolinite et attapulgite. 
La montmorillonite toujours présente, est plus abondante vers I'aval des versants et en profondeur, tandis que la proportion d'illite diminue corrélativement; la kaolinite est toujours minoritaire, quant à l'attapulgite, toujours en faible proportion, elle est liée exclusivement à la présence de marnes à gypse (semelle de nappe, diapir). Les observations faites au M.E.T. ont été confirmées par les A.T.D. A.T.P. et par spectroscopie infra-rouge.

Trois types de matériaux ont pu être caractérisés :

- un matériau à illite dominante, $W L=50$ à 63 g IP $=25$ à $33 \% ; ~$
$28 \%$ \%

- un matériau à montmorillonite dominante, wL $=77$ à 82 \% ; IP=45 à 51 \% ; o < $<2 \mu=55$ à 61 \% ; ${ }^{8} \mathrm{CaCO}_{3}=9,1$ à 12,7 \% ;

- un matériau à illite dominante avec attapulgite,

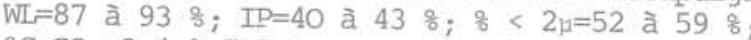
${ }_{8} \mathrm{CaCO}_{3}=3,4$ à $7,7 \%$.

Les limites sont élevées, le \& d'argiles augmentent généralement avec les limites, tandis que le \& de $\mathrm{CaCO}_{3}$ diminue. La montmorillonite est toujours présente, minéral gonflant, présentant une grande affinité pour l'eau, c'est l'élément "déstabilisant" du milieu. Le carbonate se traduit sans doute sur le plan structural par des ponts calciques (Ie Roux 1972), qui inhibent le gonflement et réduisent les limites.

\section{CONCLUSIONS}

Les réseaux de banquettes présentent de graves défauts. Au niveau de la théorie, seule la pente est prise en compte, ce qui est critiquable sur le plan de la dynamique des versants.

Au niveau de la réalisation, les réseaux de banquettes ont été utilisés sans discernement. Ainsi les terrains argileux ont été traités, or théoriquement ils n'auraient pas dûs l'être (Saccardy - 1950), car ces terrains sont sensibles à la dessication et au gonflement, parfois même les banquettes sont établies sur d'anciens glissements. Sur les pentes supérieures à $30 \%$ où le reboisement n'a pas été exécuté, la couverture d'altération fragilisée par le remaniement anthropique est mise en mouvement. La pluviométrie $(800$ à $1400 \mathrm{~mm})$ et la violence des averses facilitent les désoräres, d'autant plus que les exutoires sont dépourvus de seuils. Les réseaux de banquettes ont parfois grandement perturbé le milieu naturel ; ainsi des versants stables et boisés ont été traités, la densité du couvert végétal y est tombé de 75 à 55 \%, parfois le sol a été raclé jusqu'à la croute calcaire.

Sur le plan socio-éconornique, les banquettes constituent une entrave aux parcours et aux façons culturales traditionnelles, déclenchant l'hostilité des ruraux, ce qui explique que seuls ont été boisées les bassins versants peu peuplés.

Il apparâ̂t que seule une étude intégrée du milieu comprenant notamment : géomorphologie, géologie, géotechnique et climatologie peut rationnaliser l'utilisation des systèmes anti-érosifs.

\section{BIBLIOGRAPHIE}

Le Roux A. (1972) - Caractéristiques mécaniques des roches argileuses en relation avec leur texture.

Bull. Liaison L.P.C. $n^{\circ} 61$

Saccardy L. (1950) - Notions générales sur la lutte contre l'érosion en Algérie. Terres et Eaux, 9,51-61.

Saccaruiy L. (1950) - Notes sur le calcul des banquettes de restauration des sols. Terres et Eaux, 11,47-63. 